\title{
Early emerging probabilistic estimates govern judgments about possibility
}

\author{
Rebecca F. Schwarzlose ${ }^{1,2 *}$, Ariel Miller ${ }^{1,3}$, Elizabeth M. Williams ${ }^{1,4}$, Sabin Dang ${ }^{5}$, Lori \\ Markson $^{1}$
}

\footnotetext{
${ }^{1}$ Department of Psychological and Brain Sciences, Washington University in Saint Louis; Saint Louis, United States

${ }^{2}$ Department of Psychiatry, Washington University in Saint Louis; Saint Louis, United States

${ }^{3}$ Department of Psychological and Brain Sciences, Boston University; Boston, United States

${ }^{4}$ Applied Behavioral Analysis and Clinical Science Program, Rollins College; Winter Park, United States

${ }^{5}$ The Retina Institute; Saint Louis, United States

*Corresponding author. Email: schwarzlose@wustl.edu
}

\begin{abstract}
The complex probabilistic properties of specific events determine the number or range of possible outcomes they can produce (i.e., outcome entropy). Do humans use estimates of outcome entropy for real-world events to reason about what is possible? We test whether adults ( $N=106)$ and children $(N=368)$ use such estimates to constrain their judgments about outcomes for complex, real-world events including paint mixing and skin-color inheritance. Here we show that adults' and children's judgments reflect awareness of outcome entropy, such that fewer outcomes are deemed possible for deterministic events than probabilistic ones. Evidence of this sophisticated capacity appears between four and five years of age. Taken together, the results suggest that outcome entropy is a fundamental and early emerging factor in human reasoning about what is possible.
\end{abstract}




\section{Early emerging probabilistic estimates govern judgments about possibility}

A hallmark of human intelligence is the ability to represent complex events and their manifold possible outcomes. This crucial capacity allows us to predict the future and make inferences about unseen events in the past. Despite its practical importance, the ability to represent and reason about possibility has only recently begun to receive widespread attention from psychologists. Existing work has focused on how people generate a mental set of possible outcomes for a given event and when children demonstrate awareness that simple physical events, like a ball passing through a bifurcated tube, afford more than one possible outcome. (1-6)

Yet a fundamental feature of possibility remains unexplored in this emerging work. Probabilistic events, or events with more than one possible outcome, can differ dramatically in the number of outcomes they permit. For instance, flipping a coin yields two possible outcomes, whereas rolling a standard die affords six. We call this difference outcome entropy; events with a greater number or range of possible outcomes have higher outcome entropy than those with fewer possible outcomes. Beyond rare examples like a coin flip, the outcome entropies of most realworld events are neither explicitly taught nor precisely known and therefore can only be estimated. If individuals estimate and represent outcome entropy for a given type of real-world event, their estimates should constrain their expectations about the set of possible outcomes for that event, with potential real-world consequences. For example, people's estimates of outcome entropy for skin-color inheritance should affect their expectations about the appearance of a specific baby born to parents with specific skin colors. In turn, violations of those expectations, such as a baby born with an unexpected skin color, might cause people to reassess the initial event and question the child's paternity.

We designed a novel, color-based task to test whether adults and children use estimates of outcome entropy to constrain their judgments about possible outcomes (see Figure 1). The task is 
comprised of three within-subject conditions representing three event types: two events with high outcome entropy (i.e., fur-color inheritance for dogs and skin-color inheritance for humans) and one event with low outcome entropy (mixing two paints in equal parts to create a new color).

For each condition, we present participants with a pair of inputs (canine parents, human parents, or vials of paint) that differ in color. A story at the beginning of each condition explains that the dogs will deliver a puppy, the humans will deliver a baby, and the vials of paint will be emptied into a cup and mixed to make a new color. For each trial, an outcome probe (a puppy, baby, or cup of mixed paint) is presented beneath the input pair. The outcome probes for a given condition differ only in color; these include shades drawn from the associated color function (see Methods) and an unrelated shade (the "unrealistic trial"). For each trial, participants are asked to judge whether the presented probe could result from the input pair. The superficial structure of the three event types (i.e., two color inputs generating one color output) and the low-level features of colors presented for each event type are matched in this task, permitting direct comparison of judgments across conditions. If participants use estimates of outcome entropy to constrain their judgments of possible outcomes, they should endorse fewer probes in the paint condition than in the fur or skin conditions.

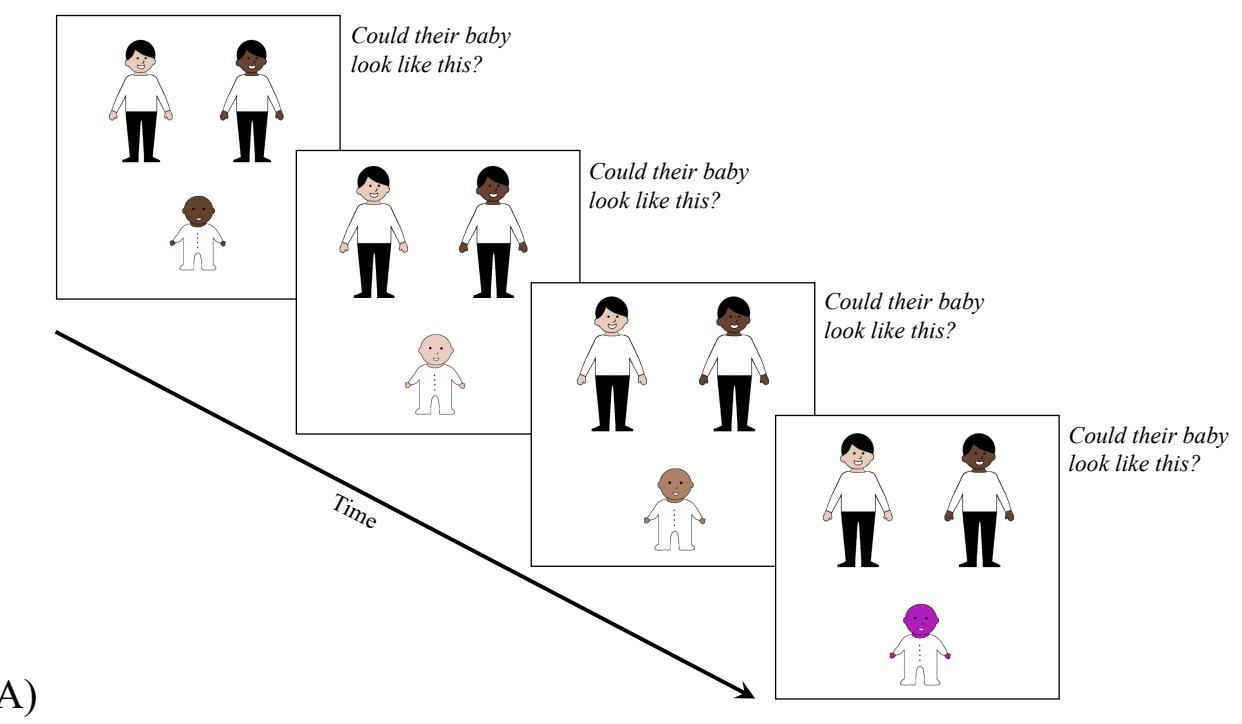


B)

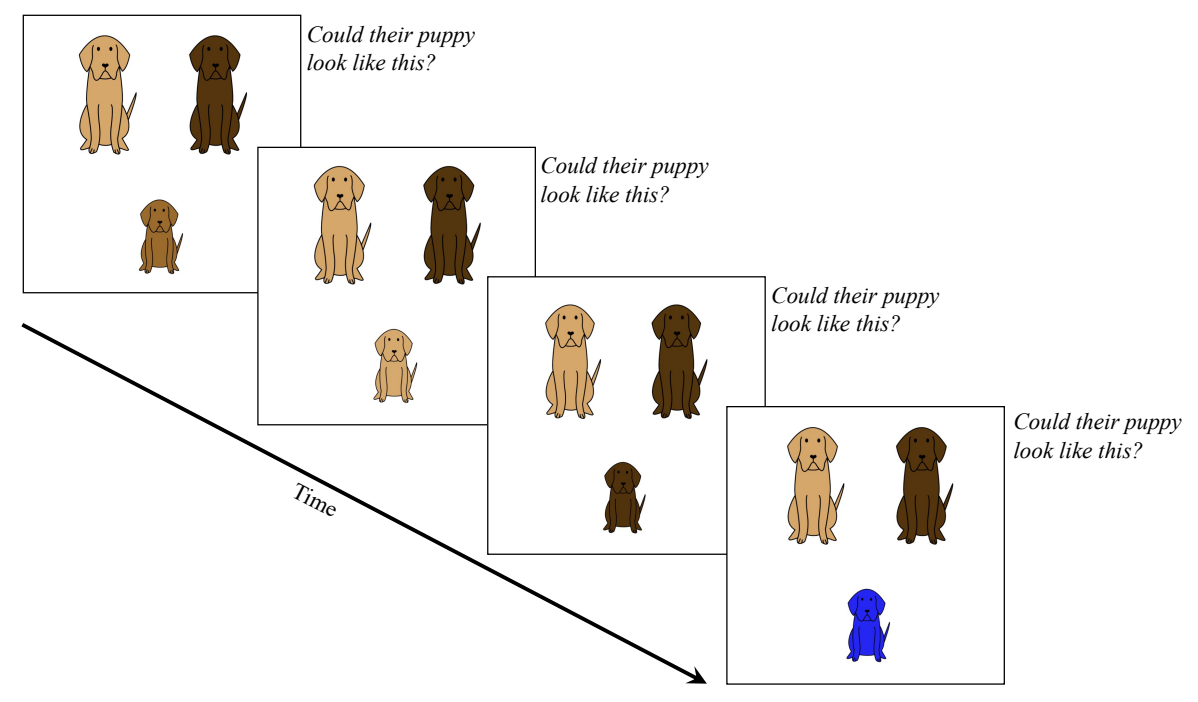

C)

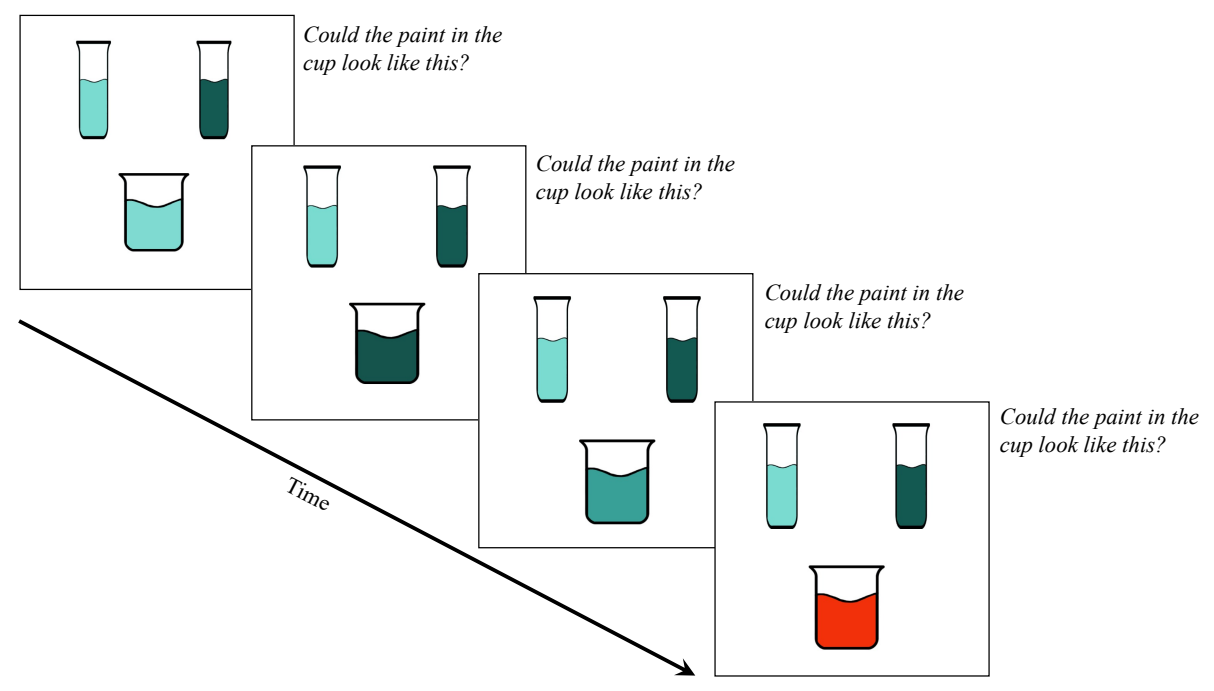

Figure 1. Examples of trials from the experiment.

Examples of individual trials, each with input pair displayed at top and output probes displayed at bottom. Examples are shown for the skin (A), fur (B), and paint (C) conditions. The unrealistic trial for each condition is shown at the far right. Trial order in the experiment was pseudorandomized.

Adults demonstrate precisely this pattern. Possible outcome counts are different across the three conditions $\left(\chi^{2}(2) \geq 115.9, p<.0005\right)$, such that possible outcome counts for paint are lower than for both fur and skin (both $p<.0005$ ). Although we anticipated that cultural and experiential factors might cause adults' estimates of outcome entropy for skin- and fur-color inheritance to 
differ, they do not $(p=1.00)$. As expected, few adults endorse unrealistic trials: $0 \%$ for the fur and skin conditions and $2 \%$ for the paint condition.

The performance of children on the same task is summarized in Figure 2. For every age group (4 to 5 years, 6 to 7 years, 8 to 9 years, and 10 years), possible outcome counts are different across the three conditions (all $\chi^{2}(2) \geq 18.8, p<.0005$ ). In each age group, post hoc pairwise comparisons reveal significant differences between possible outcome counts in the paint condition compared with both the fur and skin conditions (all $p<.03$ ), whereas the fur and skin conditions do not differ from each other (all $p>.62)$.

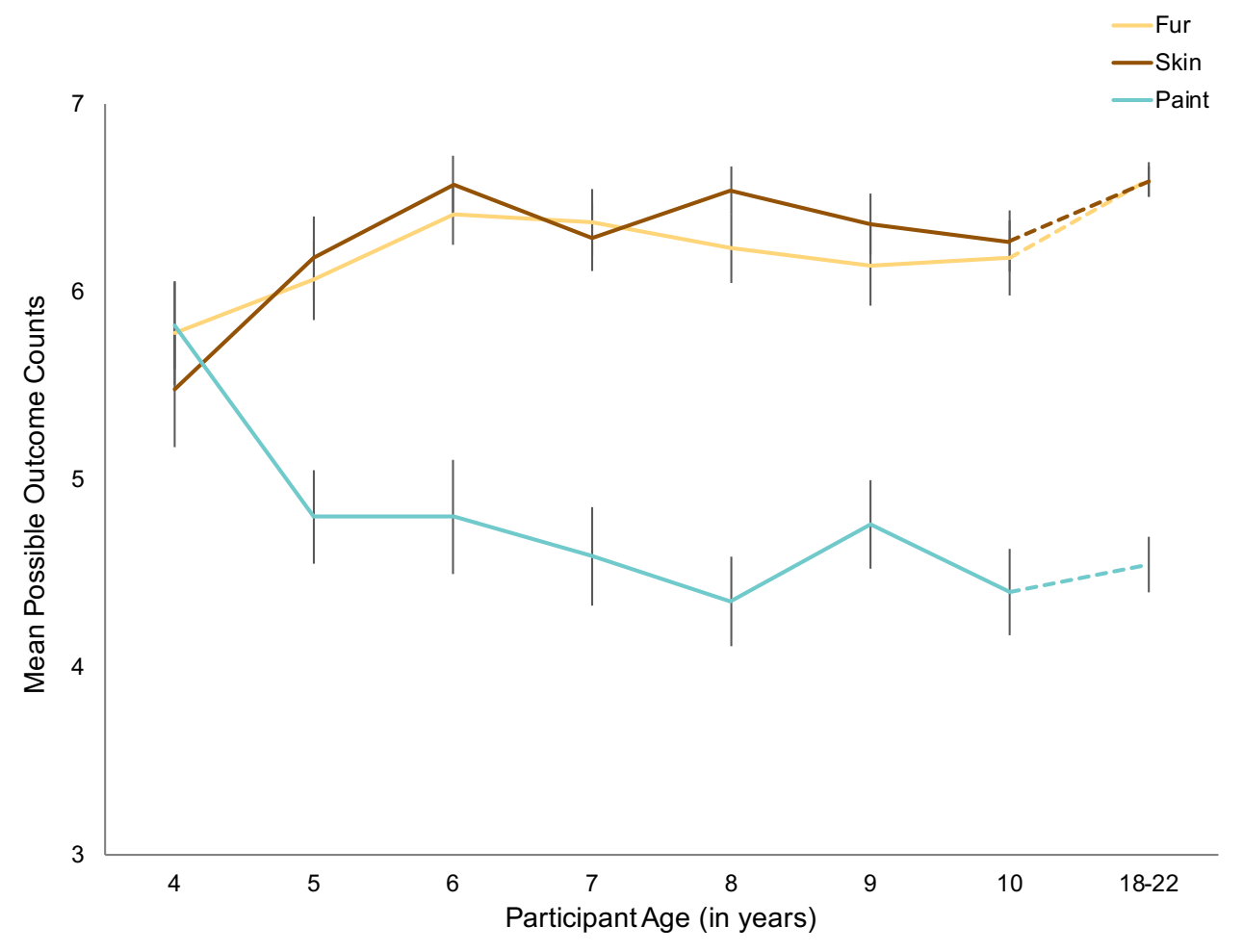

Figure 2. Possibility judgments reflect outcome entropy by 5 years of age.

Mean possible outcome counts are shown by participant age for the fur (yellow), skin (brown), and paint (turquoise) conditions. Error bars denote standard error of the mean. 
Separate analysis of 4- and 5-year-olds reveals contrasting patterns of results. Five-yearolds display the same profile as older children and adults, with different possible outcome counts across the three conditions $\left(\chi^{2}(2)=42.6, p<.0005\right)$ arising from lower possible outcome counts for paint than for skin and fur (both $p<.0005$ ). In contrast, possible outcome counts obtained from 4-year-olds do not differ across the three conditions $\left(\chi^{2}(2)=1.8, p=.42\right)$. Possible outcome counts for paint are negatively associated with age in months among 4- and 5-year-olds $(\rho=-.29, p=$ $.004)$, but not among children 6 to 10 years of age $(\rho=-.05, p=.48)$. These results suggest that $4-$ year-olds do not use estimates of outcome entropy to constrain possibility judgments, resulting in overly permissive judgments about the outcomes of low-entropy events. However, by 5 years of age, children begin to constrain judgments about low-entropy events, promoting adult-like reasoning.

The unrealistic trials provide further insight into age differences in possibility judgments. The youngest participants frequently endorse unrealistic outcomes, with $82 \%$ of 4 -year-olds and $42 \%$ of 5-year-olds endorsing at least one unrealistic probe. Endorsement of unrealistic probes in all three conditions decreases significantly with age in months among 4- and 5-year-old children (all $r_{p b}(98) \leq-.288$, all $\left.p<.005\right)$. These endorsements could indicate either that the youngest participants are unable to follow task instructions or that they make overly permissive possibility judgments. To address the former scenario, we repeated our analyses after excluding participants who endorsed one or more unrealistic probe. The pattern of results following exclusion remains the same (see supplementary text, Figure S1, Table S2).

Based on data collected from over 450 children and adults, the present findings reveal that adults and children utilize estimates of a sophisticated probabilistic feature of events - their outcome entropy - to reason about possible outcomes for real-world events, including scenarios 
with social relevance like the appearance of a biracial baby. This capacity emerges early, with robust effects of outcome entropy on 5-year-old's possibility judgments. Our results indicate that early development of this capacity, between 4 to 5 years of age, specifically allows children to constrain possibility judgments about low-entropy events, thereby narrowing down the set of possible outcomes they anticipate. This capacity could represent a major milestone in children's ability to make predictions about events in their environment and therefore capitalize upon or draw inferences from those events' outcomes.

A wealth of studies focused on counterfactual, hypothetical, and causal reasoning implicates the preschool years as a pivotal period of development in children's abilities to flexibly and simultaneously represent and consider alternative states in the past, present, or future.(6-12) Although studies using simple physical paradigms have shown that infants are sensitive to probabilistic information about their environment,(13-15) a cognitive shift appears to take place at 3 to 4 years of age that enables children to explicitly represent and prepare for two mutually exclusive possible physical outcomes simultaneously. $(4,5,16,17)$ This capacity to represent multiple possible outcomes simultaneously might be a prerequisite for the sophisticated use of outcome entropy estimates observed here in children 5 and older.

The finding that possibility judgments of 4-year-olds are more permissive than those of older children and adults, both in the low-entropy condition and in the unrealistic trials, would seem to conflict with findings that young children are more likely than adults to deem improbable events as impossible.(18-21) In the current paradigm, even unrealistic probes belong to the appropriate category; thus, young children's reasoning about possibility may show different patterns depending on whether presented outcomes differ in features versus kinds. 
In closing, the present study is the first to demonstrate that adults and children as young as 5 consider outcome entropy when reasoning about possible outcomes. The use of this sophisticated concept becomes apparent between 4 to 5 years of age and is marked by less permissive possibility judgments in low entropy scenarios. These results highlight the importance of outcome entropy for human judgments of possibility and inform future work on the development of modal, probabilistic, and predictive cognition.

\section{Materials and Methods}

\section{Participants}

Adult participants. We recruited 106 undergraduate adults through the Washington University in St. Louis psychology participant pool. Adults participated for course credit and provided written consent to participate. All adults were tested in the Cognition and Development Lab on the Washington University campus. Nine participants were excluded because of technical errors, experimenter error, participant age, participant noncompliance, or because the participant had not lived primarily in the United States. Among the remaining 97 adults (age range 18 to 22 years, $M=19.3$ years; $77 \%$ female), $53 \%$ identified as White, $14 \%$ as East Asian, $12 \%$ as Multiracial, $8 \%$ as Latinx, $7 \%$ as Black, and 5\% as South Asian.

Child participants. We recruited and tested 368 children for this study. Most child participants $(N=251)$ were recruited while visiting a free-admission science museum in the Midwest and were tested at the time of recruitment at the museum. Other participants $(N=101)$ were recruited from the community through fliers and were scheduled for testing in the Cognition and Development Lab. The remaining participants were recruited while visiting public parks and tested at the time of recruitment at the park. Parent or guardian written or verbal consent and children's verbal assent to participate were obtained prior to testing. Our pre-registered sample 
size, determined based on power analyses, was 50 children per year of age before exclusions. Data collection was prematurely halted due to the COVID-19 pandemic; therefore, samples were slightly short of this mark for the following age groups: 6 years $(N=48), 9$ years $(N=42)$ and 10 years $(N=47)$. In contrast, insufficient recruitment of 11-year-olds $(N=15)$ led us to restrict our analyses to children ages 4 through 10 years.

Of the children tested, 30 were excluded from analysis because of technical errors, experimenter error, participant age, participant noncompliance, participant illness, or because the participant had not lived primarily in the United States. Among the remaining 338 children (age range 48 to 131 months, $M=89$ months; $57 \%$ female), most (75\%) were identified as White, followed by Black (9\%), Multiracial (7\%), Latinx (3\%), and East Asian (2\%) based on parent or guardian reports.

$\underline{\text { Stimuli }}$

Color spaces. The novel task used for this study assesses reasoning about outcome entropy with respect to color properties. Therefore, input stimuli and possible outcome probes within each condition crucially differ from each other based on color. To create a systematic basis for measuring judgments within a condition and to permit valid comparisons about judgments across conditions, we created three novel color spaces. We first generated a continuous skin color space by sampling cosmetic foundation colors and extrapolating a fixed hue (22) and a continuous mathematical function for varying saturation $(S)$ and brightness $(B)$. Shades in the skin color space range from pale beige $(S=18)$ to dark brown $(S=53)$ and are defined by the following function: $H=22 ; B=\left(-.0415 S^{2}\right)+(1.4455 S)+79.48$

Analogous fur and paint color spaces are created by specifying alternate fixed hues (34 and 174, respectively) and by linearly displacing the continuous function in saturation and brightness. 
The fur color space ranges from mustard yellow $(S=48)$ to dark brown $(S=83)$ and is defined by the following function:

$H=34 ; B=\left(-.0415 S^{2}\right)+(3.93 S)-9.24$

The paint color space ranges from mint green $(S=42)$ to dark teal $(S=77)$ and is defined by the following function:

$H=174 ; B=\left(-.0415 S^{2}\right)+(3.44 S)+14.89$

The colors for all stimuli presented in the task are provided in Supplementary Table 1.

Crucially, the resulting three color spaces have obvious superficial differences between them, and yet the low-level differences among colors within each space are identical across the spaces. As a result, the task generates rigorous, carefully controlled quantitative measures of possibility judgments that permit both within- and between-subject analysis.

\section{Task Design and Measures}

The task was run online through a customized website and displayed on an iPad. The paradigm had a within-subject design, so that each participant completed all three conditions. Participants were randomly assigned to a Skin-Fur-Paint or a Skin-Paint-Fur order. This ordering was selected to prioritize the independence of the socially relevant skin color condition. Although the primacy of the skin condition could, in theory, create ordering effects on participant responses, such effects could not explain the significant differences we find in judgments between the fur and paint conditions in all but the youngest participants. In short, the pattern of results we obtained with this task cannot be an artifact of ordering effects.

Each condition began with a brief story that accompanied presentation of two input stimuli (i.e., pair of human parents, pair of dog parents, and pair of vials of paint). To protect against 
differences in input stimulus salience influencing children's predictions of event outcomes, the two input stimuli for each condition were identical to one another except for their coloration. To avoid a previously documented 'mother bias' in young children's reasoning about phenotypic inheritance, $(22,23)$ the stimuli used to represent the parents in the skin condition were designed to be gender ambiguous. The wording of the stories read for each condition was modified slightly to create age-appropriate versions of the task for adults and children. Other than these modifications, indicated in parentheses below, there were no differences between the adult and child versions of the task. For the skin condition, participants heard the following: Here is $a$ (mother/mom) and (father/dad). The (mother/mom) (is pregnant/has a baby in her tummy). They are going to the hospital to (deliver/have) their baby. What do you think their baby will look like? For the fur condition, participants heard the following: Here is a (mother/mom) dog and a (father/dad) dog. What do you think their puppy will look like? Finally, for the paint condition, participants heard the following: Here are two tubes of paint. An artist empties both tubes of paint into a cup and stirs them well. What do you think the paint in the cup will look like? The immediate prompt for every probe trial was of the form: Could look like this? The blank was filled in with their puppy, their baby, or the paint in the cup for the fur, skin, and paint conditions, respectively.

The first probe in the first (skin) condition was a purple baby for all participants. Responses to this trial were excluded from subsequent analysis. If a participant responded in the affirmative on this trial, indicating that the couple's baby could have purple skin, the experimenter gently questioned the participant about their response, asking "Have you ever seen a purple baby?" This question was intended to encourage participants to think realistically about their answers. Participants were then asked to make a judgment about the purple probe again; regardless of their 
judgment, their response was recorded and the experiment proceeded without interruption. Aside from this initial probe, experimenters did not interject or comment on participant responses.

Data presented here were collected from eight consecutive probe trials per condition. For each probe trial, participants were asked whether the probe could be the outcome of the inputs shown. For each condition, seven of these probes were realistic potential outcomes drawn from the color space generated for that condition. An eighth probe (unrealistic trial) in each condition was not drawn from the color space (i.e., a blue puppy, a magenta baby, and red paint). The ordering of probes and unrealistic trials in each condition were randomized except for the final probe, which was always the midpoint color, i.e., the color equidistant to the two input colors in its color space. The dependent variable for these analyses was possible outcome count, which was defined as the number of realistic outcome color probes the participant endorsed as possible. The experiment yielded three possible outcome counts per participant: one for each of the three conditions (fur, skin, and paint). Possible outcome counts are integer values that can range from a minimum of zero to a maximum of seven.

The task reported here was embedded within a larger study that included other types of trials and the collection of additional measures (see below for full detail). The Institutional Review Board at Washington University in Saint Louis approved all study procedures.

\section{Data Analysis}

A data analysis plan was preregistered on OSF (https://osf.io/vuxby). As described there, we ran related-samples Friedman's tests on possible outcome counts in the fur, skin, and paint conditions for each of the following age groups: ages 4 to 5 years, 6 to 7 years, 8 to 9 years, and 18 to 22 years (adults). An intended 10- to 11-year age group was not possible, due to insufficient recruitment of 11-year-olds. Therefore, we analyzed data from the 10-year-olds separately as an 
independent age group. Significant results on the Friedman's test indicating unequal distribution of scores were followed up with Bonferroni-corrected pairwise comparisons to determine which conditions were significantly different. To explore observed effects, we additionally ran independent Friedman's tests separately on 4-year-olds and 5-year-olds. We also computed Spearman's rank-order correlations between age (in months) and possible outcome count for each of the three conditions among children 4 to 5 years of age and separately, for children 6 and older. Reported $p$ values reflect two-tailed significance levels.

Given high rates of unrealistic trial endorsement among younger children, we analyzed the endorsement of these trials by age across the three conditions. Two-tailed point-biserial correlation analyses were computed to assess the relationship between endorsement of unrealistic trials and age in months among 4- and 5-year-old participants. Endorsement of unrealistic trials could indicate that a participant was unwilling or unable to follow task instructions. However, it may alternately indicate that a participant had an overly permissive judgment of what outcomes were possible. The former reason warrants exclusion of a participant's data from further analysis; the latter reason does not. To reconcile these considerations, we performed analyses both with and without exclusion of participants who endorsed any unrealistic trials; in all cases, the pattern of results was the same (see Supplementary Materials text, Figure S1). We report results from the inclusive analyses in the main text. 


\section{References}

1. J. Phillips, J. Knobe, The psychological representation of modality. Mind Lang. 33, 6594 (2018).

2. P. N. Johnson-Laird, M. Ragni, Possibilities as the foundation of reasoning. Cognition. 193, 103950 (2019).

3. J. Phillips, A. Morris, F. Cushman, How We Know What Not To Think. Trends in Cognitive Sciences. 23, 1026-1040 (2019).

4. J. Redshaw, T. Suddendorf, Children's and Apes’ Preparatory Responses to Two Mutually Exclusive Possibilities. Current Biology. 26, 1758-1762 (2016).

5. J. Redshaw, T. Suddendorf, K. Neldner, M. Wilks, K. Tomaselli, I. Mushin, M. Nielsen, Young Children From Three Diverse Cultures Spontaneously and Consistently Prepare for Alternative Future Possibilities. Child Development. 90, 51-61 (2019).

6. S. R. Beck, E. J. Robinson, D. J. Carroll, I. A. Apperly, Children's Thinking About Counterfactuals and Future Hypotheticals as Possibilities. Child Development. 77, 413-426 (2006).

7. T. McCormack, M. Ho, C. Gribben, E. O’Connor, C. Hoerl, The development of counterfactual reasoning about doubly-determined events. Cognitive Development. 45, 1-9 (2018).

8. A. Nyhout, P. A. Ganea, Mature counterfactual reasoning in 4- and 5-year-olds. Cognition. 183, 57-66 (2019).

9. A. Nyhout, P. A. Ganea, What is and what never should have been: Children's causal and counterfactual judgments about the same events. Journal of Experimental Child Psychology. 192, $104773(2020)$. 
10. A. Nyhout, L. Henke, P. A. Ganea, Children's Counterfactual Reasoning About Causally Overdetermined Events. Child Development. 90, 610-622 (2019).

11. K. Springer, F. C. Keil, Early Differentiation of Causal Mechanisms Appropriate to Biological and Nonbiological Kinds. Child Development. 62, 767-781 (1991).

12. A. Gopnik, A. Rosati, Duck or rabbit? Reversing ambiguous figures and understanding ambiguous representations. Developmental Science. 4, 175-183 (2001).

13. E. Téglás, E. Vul, V. Girotto, M. Gonzalez, J. B. Tenenbaum, L. L. Bonatti, Pure Reasoning in 12-Month-Old Infants as Probabilistic Inference. Science. 332, 1054-1059 (2011).

14. E. Teglas, V. Girotto, M. Gonzalez, L. L. Bonatti, Intuitions of probabilities shape expectations about the future at 12 months and beyond. Proceedings of the National Academy of Sciences. 104, 19156-19159 (2007).

15. N. Cesana-Arlotti, A. Martín, E. Téglás, L. Vorobyova, R. Cetnarski, L. L. Bonatti, Precursors of logical reasoning in preverbal human infants. Science. 359, 1263-1266 (2018).

16. B. P. Leahy, S. E. Carey, The Acquisition of Modal Concepts. Trends in Cognitive Sciences. 24, 65-78 (2020).

17. J. Redshaw, T. Suddendorf, Temporal Junctures in the Mind. Trends in Cognitive Sciences. 24, 52-64 (2020).

18. C. Kalish, Reasons and Causes: Children's Understanding of Conformity to Social Rules and Physical Laws. Child Development. 69, 706-720 (1998).

19. T. Kushnir, A. Gopnik, N. Chernyak, E. Seiver, H. M. Wellman, Developing intuitions about free will between ages four and six. Cognition. 138, 79-101 (2015).

20. A. Shtulman, The development of possibility judgment within and across domains. Cognitive Development. 24, 293-309 (2009). 
21. A. Shtulman, S. Carey, Improbable or Impossible? How Children Reason About the Possibility of Extraordinary Events. Child Development. 78, 1015-1032 (2007).

22. E. Engel-Clough, C. Wood-Robinson, Children's understanding of inheritance. Journal of Biological Education. 19, 304-310 (1985).

23. S. C. Johnson, G. E. A. Solomon, Why Dogs Have Puppies and Cats Have Kittens: The Role of Birth in Young Children's Understanding of Biological Origins. Child Development. 68, 404-419 (1997).

\section{Funding:}

Russell Sage Foundation grant 3122 (LM, RFS)

National Institute of Mental Health training grant MH014677-40 (RFS) 